\title{
Left main coronary artery thrombosis: An unexpected complication of venoarterial extracorporeal membrane oxygenation
}

\author{
Alexis Theron, MD, Eleonore Ravis, JD, Nicolas Jaussaud, MD, and Frederic Collart, MD, Marseille, \\ France
}

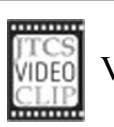

Video clip is available online.

Extracorporeal membrane oxygenation (ECMO) can be used as a bridge to decision for selected patients with mechanical complications related to myocardial infarction (MI). ${ }^{1,2}$ Here we report the case of a patient with a ventricular septal rupture (VSR) who was treated with ECMO as a bridge to stabilization before delayed surgery. Unfortunately, significant ascending aortic thrombosis with left main coronary involvement occurred despite appropriate heparin therapy.

\section{CLINICAL SUMMARY}

A 65-year-old male patient was admitted with acute respiratory failure and hypotension. The physical examination revealed signs of cardiogenic shock with an

From the Department of Cardiac Surgery, La Timone Hospital, Marseille, France. Disclosures: Authors have nothing to disclose with regard to commercial support. A.T. and E.R. contributed equally to this article.

Received for publication May 5, 2014; revisions received Aug 1, 2014; accepted for publication Aug 7, 2014; available ahead of print Sept 8, 2014

Address for reprints: Alexis Theron, MD, Department of Cardiac Surgery, La Timone

Hospital, 264, Rue St Pierre, 13385 Marseille Cedex 05, France (E-mail: alexis.

theron@ap-hm.fr).

J Thorac Cardiovasc Surg 2014; 148:e215-7

0022-5223/\$36.00

Copyright (C) 2014 by The American Association for Thoracic Surgery

http://dx.doi.org/10.1016/j.jtcvs.2014.08.009 unknown 4/6 holosystolic murmur. An electrocardiogram revealed ST elevation in the inferior leads, suggesting an inferior ST-segment elevation MI. A transthoracic echocardiogram showed a large posterior VSR with inferior left ventricular (LV) wall akinesis. Emergency coronary catheterization revealed an occlusion of the right coronary artery associated with diffuse non-significant lesions of the left anterior descending coronary artery. Despite implantation of an intra-aortic balloon pump and an increasing dose of inotropic drugs, the clinical status of the patient continued to worsen. In particular, his mean arterial blood pressure dropped below $50 \mathrm{~mm} \mathrm{Hg}$, and signs of cardiogenic shock were observed. Consequently, the patient was shifted by ground transportation to our hospital for immediate treatment with venoarterial ECMO, which was inserted surgically into the femoral vessels. The circuits were coated with heparin, and the flow through the ECMO was $4.5 \mathrm{~L} / \mathrm{min}$. Activated clotting time was 200 seconds during the ECMO implantation. Intravenous unfractionated heparin was then administered (40,000 IU/d) for a target of activated partial thromboplastin time ratio between 2 and 3. The LV was totally unloaded without pulsatility. Twenty-four hours after his admission, the patient was seen to have an anterior ST-segment elevation MI with persistent ventricular tachycardia episode despite an optimal antiplatelet therapy with aspirin $(75 \mathrm{mg}$ ) and clopidogrel bisulfate (INN clopidogrel; $75 \mathrm{mg}$ ). A second emergency coronary angiogram was performed, revealing
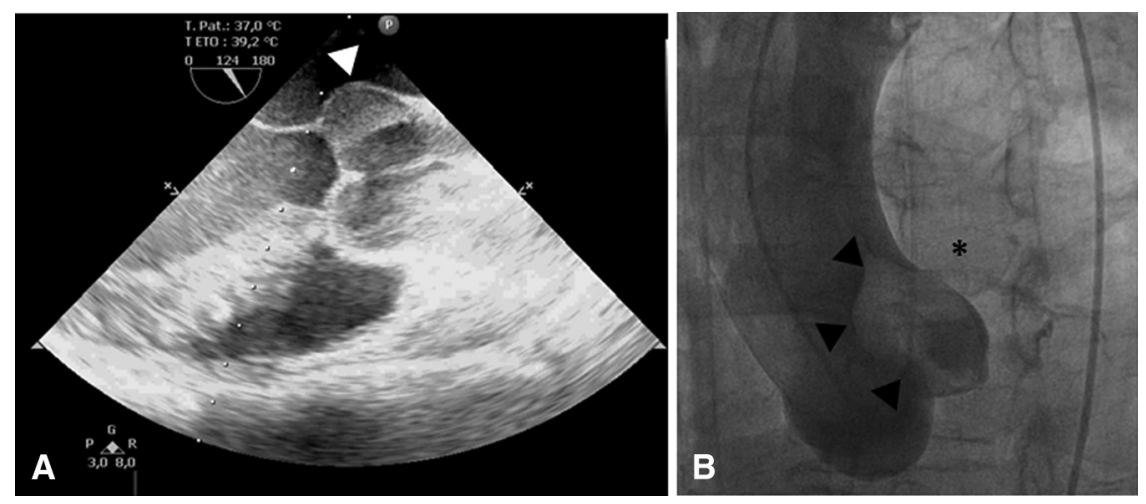

FIGURE 1. A, Long-axis 2-dimensional transesophageal echocardiographic view after the implantation of extracorporeal membrane oxygenation. The sludge in the ascending aorta indicates blood stasis in the absence of left ventricular pulsatility. The thrombus is visible in the left coronary sinus (white arrows). B, Aortography showing a markedly large thrombus of the left Valsalva sinus (black arrows) with extensive involvement of the left main coronary artery (black asterisk). The aortic valve could be seen to remain closed during diastole and systole. Interestingly, the left coronary sinus was not "washed" by the retrograde aortic flow of venoarterial extracorporeal membrane oxygenation, resulting in blood stasis and thrombus formation. 

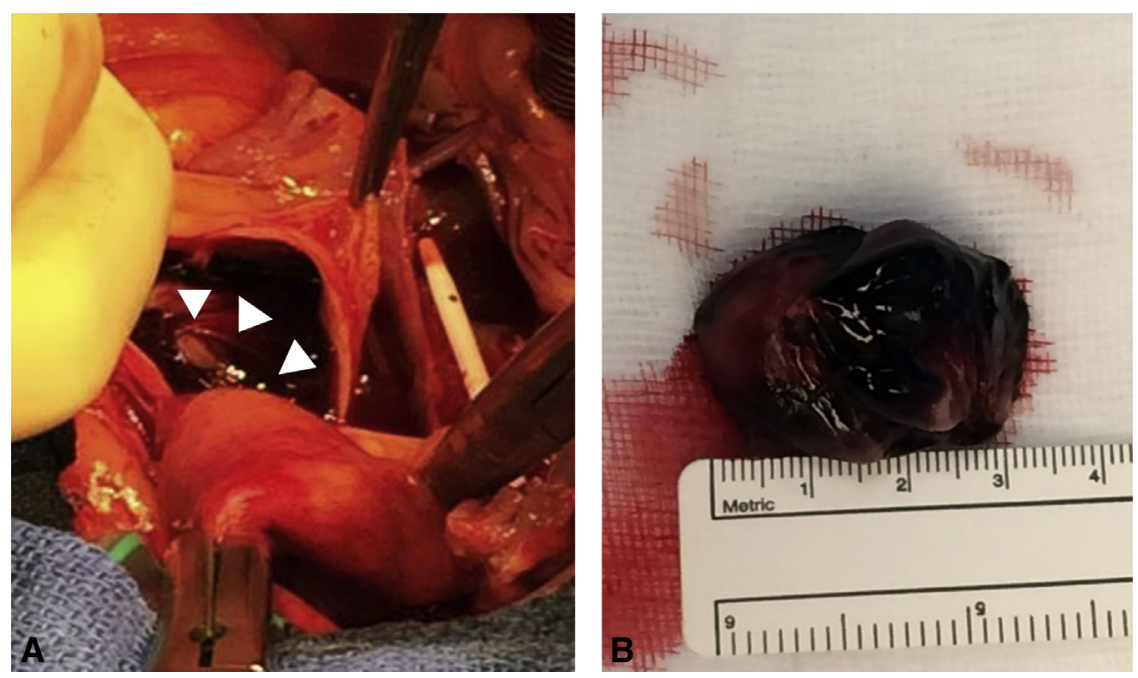

FIGURE 2. A, Perioperative photograph taken after aortotomy and before the orthotopic heart transplant. A fresh thrombus can be viewed floating in the left coronary sinus (white arrows). B, Measurement of the fresh thrombus after removal $(20 \times 30 \mathrm{~mm})$.

a large thrombus of the left Valsalva sinus with extensive involvement of the left main coronary artery that precluded coronary catheterization (Figure 1, B; Video 1). Activated partial thromboplastin time ratio was 2.3 during this complication (Figure 3). Transesophageal echocardiography confirmed these findings and revealed heterogeneous sludge in the ascending aorta corresponding to blood stasis in the absence of LV pulsatility and cardiac output (Figure 1, A). Heparin therapy was deemed the optimal course of action, because heparin-induced thrombocytopenia was ruled out by the absence of anti- platelet factor 4 antibodies. We considered the risk of surgical repair too high because of severe impairment of $\mathrm{LV}$

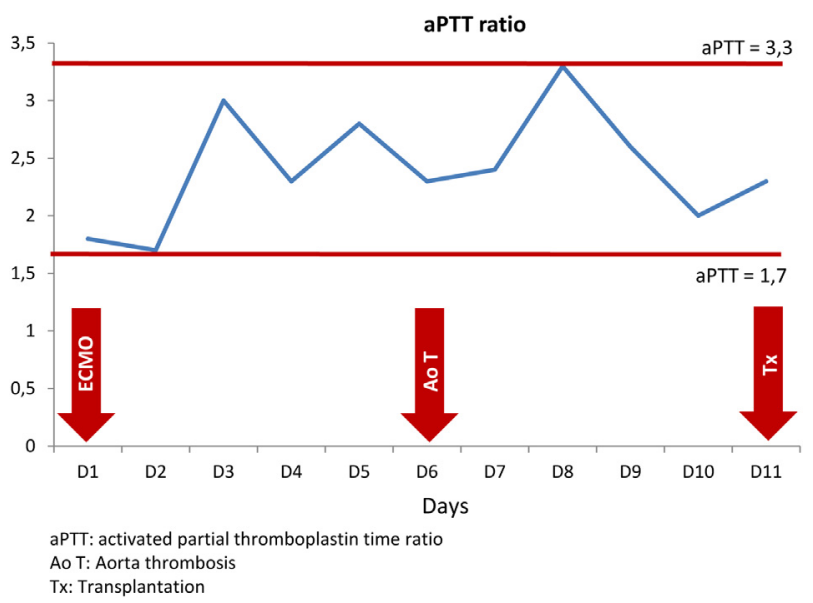

FIGURE 3. Evolution of activated partial thromboplastin time (aPTT) ratio during the patient's hospitalization in the intensive care unit. Note that aortic thrombosis (AoT) occurred despite an activated partial thromboplastin time ratio of 2.3 and an optimal antiplatelet therapy. ECMO, Extracorporeal membrane oxygenation; $T x$, orthotopic heart transplant. systolic function. Moreover, we thought that weaning from the cardiopulmonary bypass would be hazardous because of the right coronary artery and left main coronary artery thrombosis. The patient was then registered for heart transplant as an emergency procedure. Two days later, he received a heart transplant through an orthotopic approach (Figure 2). Unfortunately, the patient died 45 days later of septic shock caused by a multidrugresistant Klebsiella pneumoniae.

\section{DISCUSSION}

This is the first case report to describe a patient with extensive left main coronary thrombosis as an iatrogenic complication of $\mathrm{ECMO}^{3}$ without lack in anticoagulation. This severe condition may develop during the thrombogenic period after MI and results from blood stasis in the absence of LV pulsatility. Care should therefore be taken to maintain orthodromic cardiac output with a concomitant aortic valve opening despite an increase in the LV afterload resulting from retrograde flow in the ascending aorta. This consideration is even more relevant in cases of VSR with a left-toright shunt that decreases the LV stroke volume. In particular, care should be taken to find a balance in the ECMO output; it should be sufficient to allow organ recovery and right ventricular unloading but not so great that it fails to preserve LV ejection through the aortic valve. An intra-aortic balloon pump could be used to maintain pulsatility during ECMO support, improve coronary perfusion, and decrease the LV afterload despite the increased risk of limb ischemia. ${ }^{4}$ Moreover, enhancement with heparin therapy and modifications to the ECMO settings could be monitored with echocardiography to assess the thrombogenic risk (presence of aorta sludge or the absence of aortic valve opening). Surgical 
thrombectomy may be excluded for these critically ill patients, although an emergency transplant remains an option.

\section{CONCLUSIONS}

Coronary occlusion as a result of aortic thrombosis is a rare but catastrophic complication of venoarterial ECMO as a bridge to delayed surgery for post-MI VSR. During this highly thrombogenic period, LV pulsatility and aortic valve opening must be strictly preserved and heparin therapy closely monitored. A heart transplant should be considered in the most severe cases when the risk of surgical repair is considered too high.

\section{References}

1. Tang GHL, Malekan R, Kai M, Lansman SL, Spielvogel D. Peripheral venoarteria extracorporeal membrane oxygenation improves survival in myocardial infarction with cardiogenic shock. J Thorac Cardiovasc Surg. 2013;145:e32-3.

2. Abedi-Valugerdi G, Gabrielsen A, Fux T, Hillebrant CG, Lund LH, Corbascio M Management of left ventricular rupture after myocardial infarction solely with ECMO. Circ Heart Fail. 2012;5:e65-7.

3. Muehrcke DD, McCarthy PM, Stewart RW, Seshagiri S, Ogella DA, Foster RC et al. Complications of extracorporeal life support systems using heparin-bound surfaces: the risk of intracardiac clot formation. J Thorac Cardiovasc Surg. 1995; 110:843-51.

4. Doll N, Kiaii B, Borger M, Bucerius J, Krämer K, Schmitt DV, et al. Five-year results of 219 consecutive patients treated with extracorporeal membrane oxygenation for refractory postoperative cardiogenic shock. Ann Thorac Surg. 2004;77: 151-7; discussion 157

\title{
Mitral valve repair using autologous leaflet tissue as neochordae
}

\author{
Peter Benedikt, MD, MSc, ${ }^{a}$ Christian Punzengruber, MD, ${ }^{\mathrm{b}}$ Laszlo Milassin, MD, ${ }^{\mathrm{a}}$ and \\ Choi Keung Ng, MD, ${ }^{c}$ Wels and Innsbruck, Austria
}

Since the introduction of standardized mitral valve repair, techniques have continuously evolved. Different methods are known to avoid or minimize tissue resection, like artificial chordal replacement or autologous pericardial leaflet augmentation. ${ }^{2}$ We report the case of a patient who underwent mitral valve repair 20 years ago using autologous tissue leaflet as neochordae according to a technique of Gregory and colleagues. ${ }^{3}$

From the Department of Cardio, Thoracic, and Vascular Surgery, ${ }^{\mathrm{a}}$ General Hospital Wels, Wels, Austria; Division of Cardiology, ${ }^{\mathrm{b}}$ Department of Internal Medicine, General Hospital Wels, Wels, Austria; and Private Practice, ${ }^{\mathrm{c}}$ Innsbruck, Austria.

Disclosures: Authors have nothing to disclose with regard to commercial support.

Received for publication July 30, 2014; accepted for publication Aug 29, 2014; available ahead of print Sept 22, 2014.

Address for reprints: Peter Benedikt, MD, MSc, Department of Cardio, Thoracic, and Vascular Surgery, General Hospital Wels, Grieskirchnerstrasse 42, 4600 Wels,

Austria (E-mail: peter.benedikt@klinikum-wegr.at).

J Thorac Cardiovasc Surg 2014;148:e217-8

$0022-5223 / \$ 36.00$

Copyright (C) 2014 by The American Association for Thoracic Surgery

http://dx.doi.org/10.1016/j.jtcvs.2014.08.047

\section{CLINICAL SUMMARY}

A 52-year-old man was admitted to our hospital for severe regurgitation with prolapse and chordal rupture at the posterior leaflet of a myxomatous mitral valve. Coronary angiography results and left ventricular function were normal.

\section{SURGICAL TECHNIQUE}

After median sternotomy cardiopulmonary bypass was set up by bicaval cannulation. Heart arrest was achieved with cold crystalloid cardioplegia. The mitral valve was exposed via right atriotomy through the interatrial septum.

After mitral valve analysis, which depicted a flail leaflet in $\mathrm{P} 2$ and accompanying prolapse in $\mathrm{P} 1$ and $\mathrm{P} 3$, the valve was repaired by using a strip of autologous tissue of the posterior mitral leaflet as neochordae.

Two parallel incisions at a distance of $4 \mathrm{~mm}$ were set in the prolapsing segment of the posterior mitral leaflet
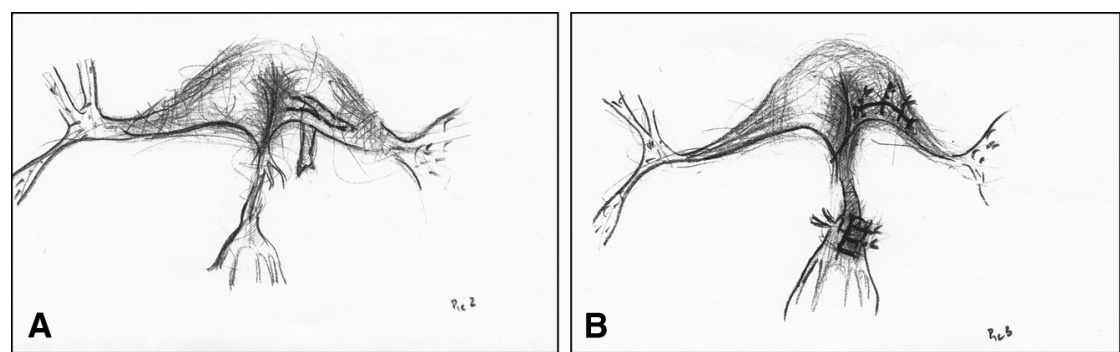

FIGURE 1. A, Two parallel incisions at a distance of $4 \mathrm{~mm}$ are set in the prolapsing segment of the posterior mitral leaflet beginning $2 \mathrm{~mm}$ from the annulus and ending $3 \mathrm{~mm}$ before the free edge. B. The excised strip is anchored to the papillary muscle with pericardial pledgeted-supported 5-0 polypropylene sutures. 